Document downloaded from:

http://hdl.handle.net/10251/82770

This paper must be cited as:

Antonino Daviu, E.; Mohamed Mohamed-Hicho, N.; Cabedo Fabres, M.; Ferrando Bataller, M. (2016). Modal pattern stability factor as a figure of merit for characteristic modes.

Electronics Letters. 52(20):1658-1659. doi:10.1049/el.2016.1926.

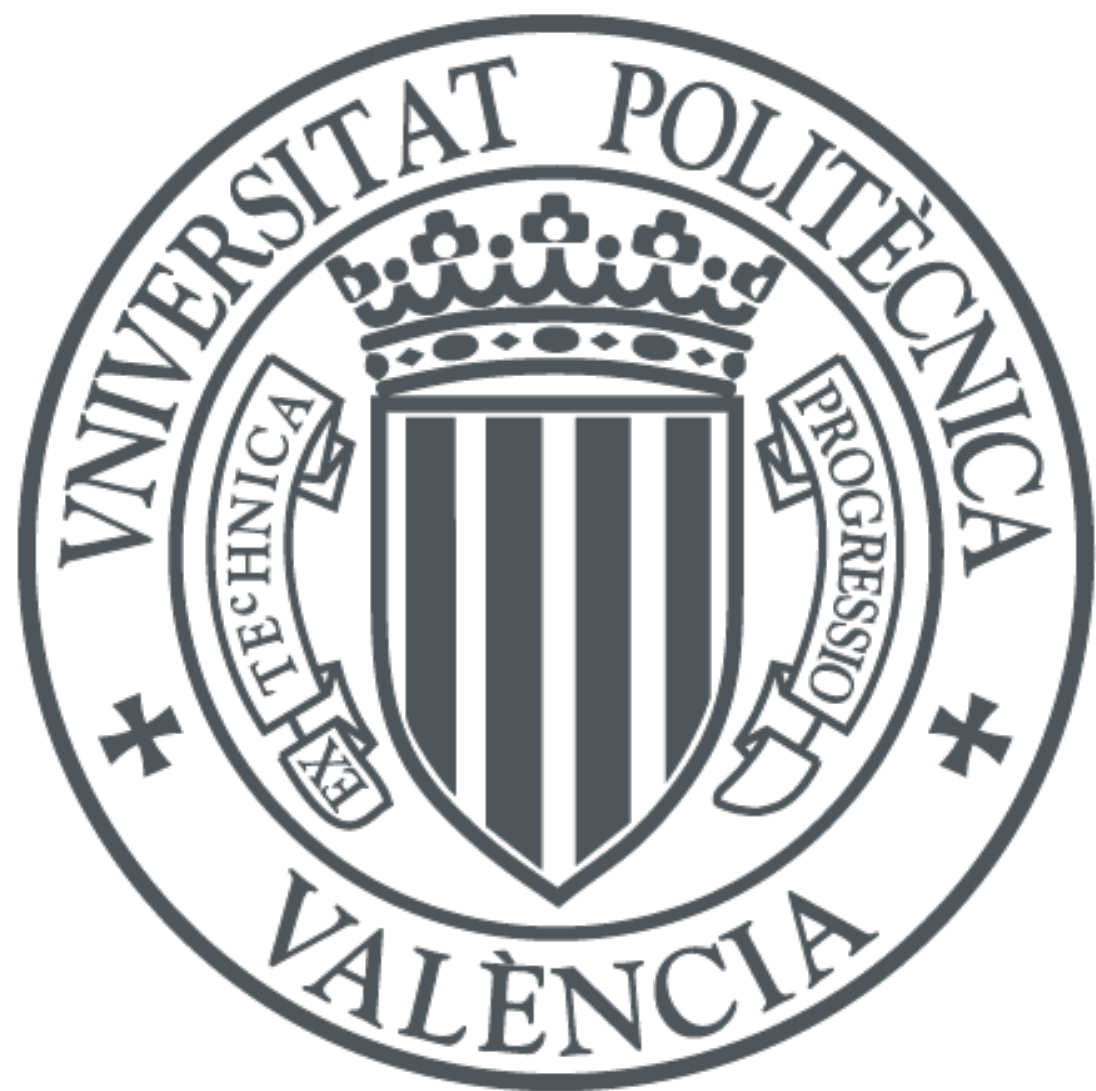

The final publication is available at

http://dx.doi.org/10.1049/el.2016.1926

Copyright Institution of Engineering and Technology (IET)

Additional Information

This paper is a postprint of a paper submitted to and accepted for publication in Electronics Letters and is subject to Institution of Engineering and Technology Copyright. The copy of record is available at the IET Digital Library. 


\section{Modal Pattern Stability Factor as a Figure of Merit for Characteristic Modes}

E. Antonino-Daviu, N. Mohamed Mohamed-Hicho, M. Cabedo-Fabrés and M. Ferrando-Bataller

This letter proposes the use of a Modal Pattern Stability Factor (MPSF) parameter to quantify the pattern stability associated to Characteristic Modes (CM). The proposed parameter can be used as a figure of merit for $\mathrm{CM}$ associated to arbitrary-shaped structures, aiding the antenna design process. This parameter can be used (together with other modal parameters) to optimize the geometry of a radiating element in order to improve the radiation pattern stability in a desired range of frequencies. Two examples are presented for analysis. First, MPSF for the CM of three ring antennas with different width are analysed, showing how pattern stability of CM depend on this geometrical parameter. Secondly, three metallic plates with different shapes are analysed in terms of MPSF, in order to assess the stability of the CM for each geometry.

Introduction: The Theory of Characteristic Modes described by Harrington and Mautz in [1] defines a set of real modes on the surface of a conducting or dielectric body, which can be used to expand the total current. These modes are independent of any kind of excitation and depend only on the shape and size of the conducting object. In the last decade, CM have attracted increasing interest for antenna design [2], as they provide physical insight into the radiation behaviour of antennas. Different parameters have been investigated and proposed lately in order to quantify the information provided by CM, like modal Q-factor or modal radiation efficiency.

Radiation pattern associated to CM changes with frequency, depending on the intrinsic electrical properties of the radiating structure. In this paper, we propose a new parameter that aims to compute the stability of the radiation pattern associated to a specific CM. This parameter, called Modal Pattern Stability Factor (MPSF), is based in the Pattern Stability Factor parameters (PSF and PSFII) defined in [3] and [4], with the purpose of quantifying the stability of the radiation pattern associated to wideband antennas for a specific bandwidth and range of directions. Here, a similar parameter is proposed for $\mathrm{CM}$, in order to assess the stability with frequency of the radiation pattern associated to the $\mathrm{CM}$ of any structure, providing a new figure of merit for $\mathrm{CM}$ which can certainly help in the antenna design process. This parameter can be used (together with other modal parameters) to optimize the geometry of a radiating element in order to obtain radiation pattern stability in a desired range of frequencies.

Definition of MPSF will be presented next, and two cases will be analysed hereinafter in order to show the interest of the proposed parameter.

Modal Pattern Stability Factor (MPSF): Equation (1) presents the autocorrelation factor $\rho_{n}$ in the frequency domain of the 3D radiation pattern associated to the $n^{\text {th }} \mathrm{CM}$ with respect to a reference frequency $f_{R}$ :

$$
\rho_{n}\left(f, f_{R}\right)=\frac{\left|\oint_{4 \pi}\left[e_{n}(f, \Omega) \cdot e_{n}^{*}\left(f_{R}, \Omega\right)\right] \cdot d \Omega\right|^{2}}{\int_{4 \pi}\left|e_{n}(f, \Omega)\right|^{2} d \Omega \cdot \int_{4 \pi}\left|e_{n}\left(f_{R}, \Omega\right)\right|^{2} d \Omega}
$$

where $e_{n}(f, \Omega)$ is the $3 \mathrm{D}$ radiated electric far field of mode $n$ at frequency $f$, and $d \Omega=\sin \theta \cdot d \theta \cdot d \phi$. Similarly to [4], the average value of the correlation factor between the radiated modal electric field in reference frequency $f_{R}$ is given by

$$
C_{n}\left(f_{R}, x\right)=\frac{\left[\int_{f} \rho_{n}\left(f, f_{R}\right) \cdot d f\right]}{\left[\int_{f} d f\right]}
$$

Finally, the average value of $C_{n}$ gives the $P S F I I_{n}[4]$ or $M_{P S F}$ :

$$
\operatorname{MPSF}_{n}=\frac{\left[\int_{f} C_{n}(f) \cdot d f\right]}{\left[\int_{f} d f\right]}
$$

MPSF in ring antennas: In this first analysis, the stability of the CM associated to three circular rings with different width $(w)$ will be investigated. Fig. 1 shows the three structures under analysis, whose width goes from $2 \mathrm{~mm}$ (wire loop) to $30 \mathrm{~mm}$. In all three cases the internal radius is $R=26 \mathrm{~mm}$.

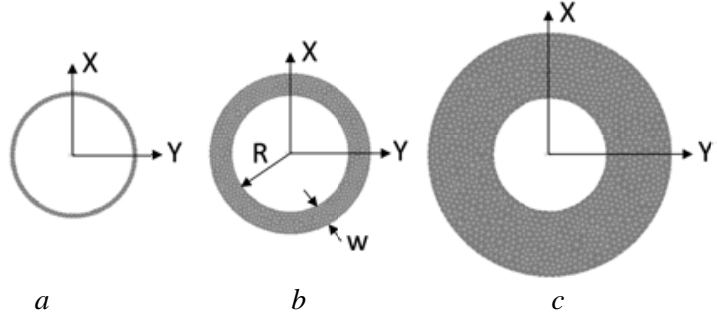

Fig. 1 Circular ring with different widths (w): a) $w=2 \mathrm{~mm}$; (b) $w=10$ $\mathrm{mm}$ and c) $w=30 \mathrm{~mm}$. Radius is $R=26 \mathrm{~mm}$ for all cases.

As known, CM associated to wires or narrow plates present narrowband behaviour or high Q-factor [2]. The wider the structure is, the larger the bandwidth (the lower the Q-factor) the CM present and hence wideband antennas can be obtained with proper excitation. However, their pattern stability varies in a different manner, what can be easily computed with the proposed MPSF parameter.

Fig. 2 shows the radiation pattern variation with frequency associated to the first $\mathrm{CM}\left(J_{1}\right)$ for rings with different widths. As observed, radiation pattern changes with the electrical length for all three structures, showing a different degree of variation in each structure for the same mode $J_{1}$. Fig. 3 shows the pattern autocorrelation vs. frequency for mode $J_{1}$. Current distribution associated to mode $J_{1}$ is also shown in the figure. Considering the resonance frequency of the mode as the reference frequency $\left(f_{R}=f_{\text {res }}\right)$ in equation (1), it can be observed that thinner rings $(w=2 \mathrm{~mm})$ presient more stable radiation patterns for mode $J_{1}$ than wider rings. Resonance frequency $\left(f_{\text {res }}\right)$ of mode $J_{1}$ is remarked in Fig. 2 for each structure.

Table 1 shows the Q-factor and MPSF associated to mode $J_{1}$ for the three analysed structures. As observed, an increase of the ring width leads to a lower Q-factor for mode $J_{1}$, but at the same time, stability for the modal radiation pattern is degraded, as MPSF decreases for this mode. In this case, bandwidth considered for the analysis is from 0 to 5 $\mathrm{GHz}$, but other bandwidth can be considered depending on the frequency range of interest.

Finally, Fig. 4 shows the autocorrelation obtained for mode $J_{4}$ (which resonates at higher frequencies) in each of the three structures. Current distribution of mode $J_{4}$ is also shown in the figure. Table 1 shows the modal Q-factor for mode $J_{4}$ and its MPSF up to $8 \mathrm{GHz}$. For this mode, the behaviour shown by the pattern stability is opposite to the previous case, as in this case stability is improved for wider rings.

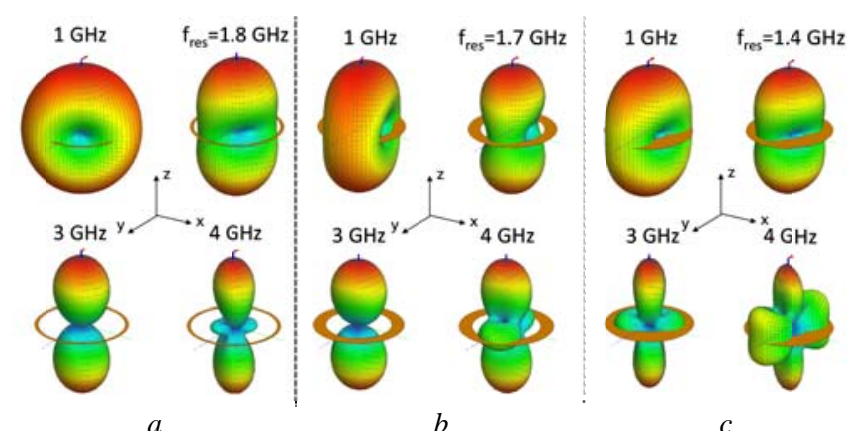

Fig. 2 Radiation pattern associated to the first $C M\left(J_{1}\right)$ of a circular loop with different widths: a) $w=2 \mathrm{~mm}$; (b) $w=10 \mathrm{~mm}$ and c) $w=30 \mathrm{~mm}$. 


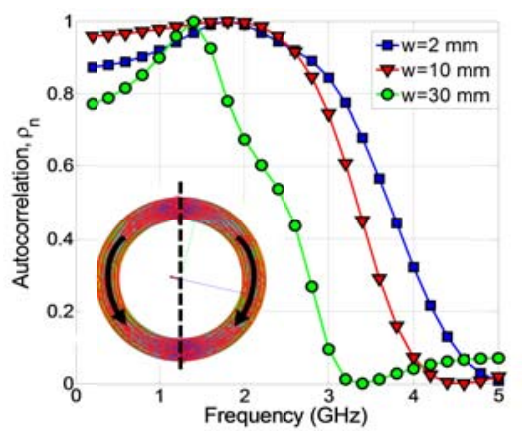

Fig. 3 Radiation pattern autocorrelation $\left(\rho_{n}\right)$ vs. frequency for the first $C M\left(J_{1}\right)$ associated to a circular ring with different widths, and current distribution of mode $J_{1}$ (dotted line marks the nulls of $J_{1}$ ).

Table 1: Modal parameters for the rings with different widths ( $w$ ).

\begin{tabular}{|c|c|c|c|c|}
\hline Width & $\begin{array}{c}\text { Q-factor } \\
\left(\mathrm{Q}_{1}\right)\end{array}$ & $\begin{array}{c}\mathrm{MPSF}_{\mathrm{n}=1} \\
(\mathrm{BW}=0-5 \\
\mathrm{GHz})\end{array}$ & $\begin{array}{c}\mathrm{Q}-\text {-factor } \\
\left(\mathrm{Q}_{4}\right)\end{array}$ & $\begin{array}{c}\mathrm{MPSF}_{\mathrm{n}=4} \\
(\mathrm{BW}=0-8 \\
\mathrm{GHz})\end{array}$ \\
\hline $\begin{array}{c}\text { Wire loop } \\
(w=2 \mathrm{~mm})\end{array}$ & 4.256 & 0.610 & 5 & 0.334 \\
\hline$w=10 \mathrm{~mm}$ & 1.803 & 0.557 & 2.087 & 0.444 \\
\hline$w=30 \mathrm{~mm}$ & 0.847 & 0.398 & 0.486 & 0.426 \\
\hline
\end{tabular}

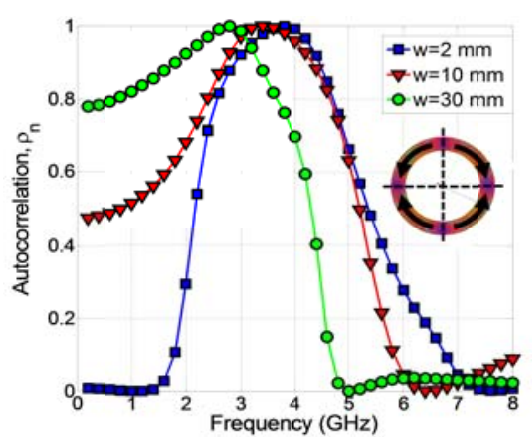

Fig. 4 Radiation pattern correlation $\left(\rho_{n}\right)$ vs. frequency for mode $J_{4}$ associated to a circular ring with different widths, and current distribution of mode $J_{4}$ (dotted lines mark the nulls of $J_{4}$ ).

Metallic Plates: In this case, the MPSF will be analysed for planar metallic plates with different shapes and the same length. Fig. 5 shows the three structures analysed: a square plate of length $L=52 \mathrm{~mm}$, a similar plate with truncated corners and a circular plate of diameter $\mathrm{L}$ $(R=L / 2=26 \mathrm{~mm})$.

Fig. 6 shows the radiation pattern variation with frequency for the first CM $\left(J_{1}\right)$ of the plates. This mode presents a vertical current distribution along the structure, as shown in [2]. Again, the variation is different depending on the geometry of the plate. Fig. 7 shows the autocorrelation obtained for mode $J_{1}$ considering the resonance frequency of the mode as the reference frequency $\left(f_{R}=f_{\text {res }}\right)$, for the three planar structures. Table 2 shows the Q-factor and MPSF computed for mode $J_{1}$ in the three plates. As seen, the square plate presents the lowest bandwidth and the lowest pattern stability for mode $J_{1}$, whereas the circular geometry leads to the best stability and bandwidth in this case.

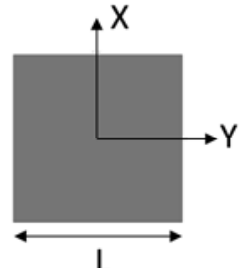

$a$

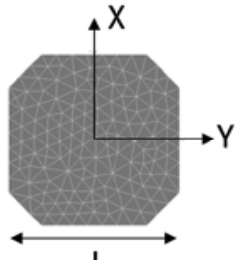

$\mathrm{L}$

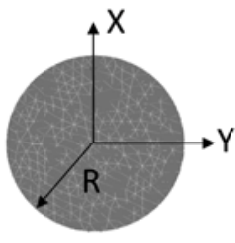

$c$

Fig. 5 Metallic plates with different shapes: a) Square plate with $L=52$ $\mathrm{mm}$; b) Square plate with $L=52 \mathrm{~mm}$ and truncated corners; c) Circular plate with $R=L / 2=26 \mathrm{~mm}$.

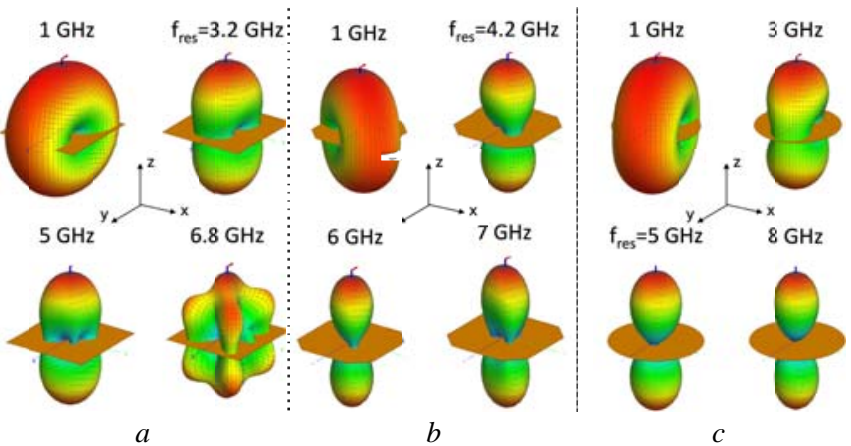

Fig. 6 Variation with frequency of the radiation pattern associated to the first $C M\left(J_{1}\right)$ of the structures shown in Fig. 5: a) Square plate; b) Square plate with truncated corners; c) Circular plate.

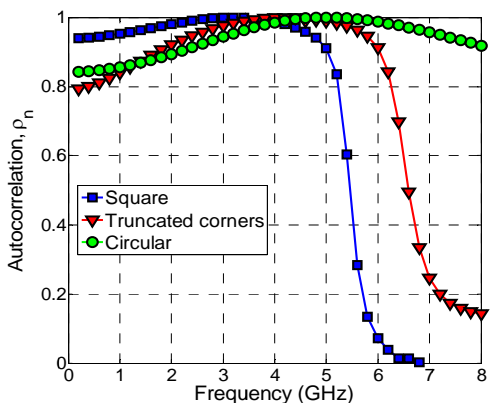

Fig. 7 Radiation pattern correlation vs. frequency for the first $C M\left(J_{1}\right)$ associated to the structures shown in Fig. 5.

Table 2: Modal parameters for the plates shown in Fig. 5.

\begin{tabular}{|c|c|c|}
\hline Plate geometry & $\begin{array}{c}\mathrm{Q}-\mathrm{factor} \\
\left(\mathrm{Q}_{1}\right)\end{array}$ & $\begin{array}{c}\mathrm{MPSF}_{\mathrm{n}=1} \\
(\mathrm{BW}=0-7 \mathrm{GHz})\end{array}$ \\
\hline Square & 0.228 & 0.6304 \\
\hline Truncated corners & 0.0697 & 0.8476 \\
\hline Circular & 0.001 & 0.9349 \\
\hline
\end{tabular}

Conclusion: Modal Pattern Stability Factor (MPSF) parameter is proposed as a figure of merit for CM, in order to perform a quantitative measure of the pattern stability associated to each mode. The proposed parameter can be used in arbitrary-shaped structures to compare the suitability of different antenna geometries for radiation pattern stability and/or to assess pattern stability improvement methods.

Acknowledgments: This work was supported by the Spanish Ministry of Economics and Competitiveness under project TEC2013-47360-C3-3P, and by Generalitat Valenciana under project GV/2015/065.

E. Antonino-Daviu, N. Mohamed, M. Cabedo-Fabrés, and M. FerrandoBataller (iTEAM of the Universitat Politècnica de València, c/ Camino de Vera s/n, 46022 Valencia, Spaim). E-mail: evanda@upvnet.upv.es

\section{References}

1 R. F. Harrington and J. R. Mautz, "Theory of Characteristic Modes for Conducting Bodies", IEEE Trans. Antennas and Propag., AP-19, 5, pp. 622-628, Sep. 1971.

2 M. Cabedo-Fabres, E. Antonino-Daviu, A. Valero-Nogueira and M. Ferrando-Bataller, "The Theory of Characteristic Modes Revisited: A contribution to the design of antennas for modern applications", IEEE Antennas and Propag. Mag., vol. 49, No. 5, pp.: 52 - 68. Oct. 2007. 3 T. Dissanayake, and K.P. Esselle, "Correlation-Based Pattern Stability Analysis and a Figure of Merit for UWB Antennas", IEEE Trans. Antennas and Propag., vol. 54, 11, pp. 3184-3191, Nov. 2006. 4 F. Fereidoony, S. Chamaani, and S.A. Mirtaheri, "UWB Monopole Antenna with Stable Radiation Pattern and Low Transient Distortion”, IEEE Antennas and Wireless Propag. Let., vol. 10, pp. 302-305, 2011. 